\section{Correlation between MTHFR Genotype Polymorphisms and Different Types of Migraine Headache in Iranian Patients: A Case Control Study}

\section{Abstract}

Background: Migraine affects about $16 \%$ of the worldwide population. The pathogenesis of the disease consists of a combination of genetic and environmental factors. Given that, according to different polymorphism studies, ethnicity has a role in the gene diversity of migraine, this study was designed to determine the role of MTHFR C677T gene polymorphism in people from Tehran, Iran, who suffer from migraine headaches.

Materials and methods: In this case control study, we determined the prevalence of common MTHFR C677T polymorphism in 132 migraine patients in line with IHS criteria (37 migraine patients with aura and 95 migraine patients without aura) and compared it to that in 142 matched healthy controls. The correlation between migraine and genotype was investigated using the chi-square test. The Bonferroni adjusted P-value was also used for pairwise comparison.

Results: Our findings demonstrated that there was no significant difference in the frequencies of the TT genotype or T allele distributions between the migraine patients with aura and the control subjects. On the other hand, the frequency of the TT genotype among migraine patients without aura was significantly lower than those in the control group or the patients with aura group.

Conclusion: MTHFR C677T polymorphism, which is responsible for reducing MTHFR activity in the folate metabolism, is not a major genetic susceptibility factor for migraine in the investigated population.

Keywords: MTHFR C677T gene; Polymorphism; Migraine; SNP

Received: September 06, 2017; Accepted: October 03, 2017; Published: October 09, 2017

\section{Introduction}

Migraine is a moderate-to-severe headache, which can be felt as a recurring throbbing pain on one side of the head. Many migraineurs also present symptoms, such as nausea, vomiting and increased sensitivity to light or sound. Migraine is a common neurovascular disorder affecting around one in every five women and around one in every 15 men, which usually begins in early adulthood. There are several types of migraine, including migraine with aura, where there are specific warning signs immediately before the migraine begins, and migraine without aura (the most common type), where the migraine occurs without any specific warning sign [1].

\author{
Reza Mirfakhraie', \\ Khosro Jamebozorgi ${ }^{2}$, \\ Mahtalb Ramezani ${ }^{3}$, \\ Farhad Assarzadegan ${ }^{3}$, \\ Omid Hesami ${ }^{3}$, \\ Marjan Asadollahi ${ }^{3}$ and \\ Behnam Safarpour Lima ${ }^{3 *}$
}

*Corresponding author:

Behnam Safarpour Lima

§ drbehnam_safarpour@yahoo.com

Department of Neurology, Shahid Beheshti University of Medical Sciences, Tehran, Iran.

Tel: 00982173430

Citation: Mirfakhraie R, Jamebozorgi K, Ramezani M, Assarzadegan F, Hesami O, et al. (2017) Correlation between MTHFR Genotype Polymorphisms and Different Types of Migraine Headache in Iranian Patients: A Case Control Study. J Neurol Neurosci. Vol.8 No. S4: 228 
the corresponding enzyme. The enzyme dysfunction is effective in the pathology of some diseases, including migraine. Many previous studies have reported that the MTHFR C677T gene polymorphism and the TT genotype are related to migraine. However, several lines of evidence have failed to accurately confirm the association between MTHFR C667T polymorphism and migraine $[5,6]$. Thus, the role of MTHFR C677T gene polymorphism in migraine susceptibility needs further evaluation.

According to different polymorphism studies, ethnicity has a role in the gene diversity of migraine. Therefore, the aim of the present study was to investigate the role of MTHFR C677T gene polymorphism in people from Tehran, Iran, who suffer from migraine. In this study, two types of migraine (with and without aura) were analyzed separately as well. Since the association between MTHFR C677T gene polymorphism and migraine is a highly controversial issue, we only evaluated this polymorphism in the MTHFR gene.

\section{Materials and Methods}

In this case control study, 132 patients with migraine (with and without aura) were selected from among people who were referred to the neurology clinics in the Imam Hossein and Loghman-e-Hakim Hospitals, according to ICHD III criteria (The International Classification of Headache Disorders: Second Edition. Cephalalgia. 2004; 24, Suppl. 1:9-160). Along with neurological examination and brain imaging, detailed information about the presence of headache and its characteristics, such as severity, frequency, duration, location, type, aura symptoms and associated symptoms (nausea, vomiting, photosensitivity or photosensitivity), were collected on a data sheet. The age range of the selected patients was 18 to 70 years. Patients with major depression and psychotic disorders were excluded from the study.

Meanwhile, from those without any history of any neurological disorders, and following a physical examination, 142 healthy controls were enrolled. The two groups were matched with regard to age and sex.

After obtaining informed consent from case and control members participating in the project, a $2.5 \mathrm{ML}$ blood sample was taken from each via a sample tube containing EDTA.

To conduct the relevant tests, the samples were sent to the Genetics Laboratory of the School of Medicine at the Shahid Beheshti University of Medical Sciences. After extraction of DNA, the tetra-primer ARMS-PCR method was briefly used for the genotyping and allele frequencies.
In the present study, the design of forward and reverse primers was performed using appropriate software and websites, such as NCBI Primer-BLAST, Gene Runner and Primer 1.

The correlation between polymorphism and the disease was evaluated with SNPStats and SNPAnalyzer, while the one between migraine and genotype was investigated by using the chi-square test.

\section{Results}

The enrolled participants comprised 142 (51\%) healthy people (73 women and 69 men) and 132 (49\%) patients (67 women and 65 men). Among the migraineurs, 95 (71.9\%) were without aura and 37 (28.1\%) were with aura. There were no significant differences in the mean age of both groups.

Of those with migraine, the location of the pain was unilateral in 68 people (51.5\%), while 64 people (48.5\%) experienced bilateral headache. There were 116 people $(87.8 \%)$ who experience nausea and vomiting, while the rest (12.2\%) did not have these conditions.

Of the 274 people who were evaluated for genotype, 152 (55.5\%) were of the CC type, 100 (36.5\%) were of the CT type and 22 (8\%) were of the TT type.

Among those who suffered from migraine, $50 \%$ were of the CC type, $44.7 \%$ were of the CT type and $5.3 \%$ were of the TT type. These ratios among healthy persons were $60.6 \%, 28.9 \%$ and $10.6 \%$, respectively. Table 1 displays frequency of MTHFR genotype variants in the patient and control groups.

It is evident from the information provided in Table 1 that the $\mathrm{TT}$ genotype and the T allele frequency are significantly lower in those who experience migraine without aura than those who experience migraine with aura and those in the control group $(p=0.026)$.

The results of the Bonferroni adjusted P-value are shown in Table 2. As it can be observed, there is a significant correlation between genotype and healthy people or those who experience migraine without aura $(p=0.015)$.

Among patients without aura, $49.5 \%$ were of the CC type, while $60.6 \%$ of healthy people had this kind of genotype.

Pairwise comparison of the other groups showed no significant correlation between the migraine with aura group and the migraine without aura group.

Table 1 Correlation between the studied groups and genotype.

\begin{tabular}{|c|c|c|c|c|c|c|c|c|}
\hline \multirow{4}{*}{ Group } & \multicolumn{7}{|c|}{ Genotype } & \multirow{3}{*}{$P$ value } \\
\hline & \multicolumn{3}{|c|}{ CC } & \multicolumn{2}{|c|}{ CT } & \multicolumn{2}{|c|}{$\mathrm{TT}$} & \\
\hline & \multicolumn{2}{|c|}{ Count } & (Percent) & Count & (Percent) & Count & (Percent) & \\
\hline & without aura & 47 & (49.5\%) & 45 & (47.4\%) & 3 & (3.2\%) & \multirow{3}{*}{0.026} \\
\hline & with aura & 19 & (51.4\%) & 14 & (37.8\%) & 4 & $(10.8 \%)$ & \\
\hline & control & 86 & (60.6\%) & 41 & $(28.9 \%)$ & 15 & (10.6\%) & \\
\hline
\end{tabular}


Table 2 Correlation between genotype in healthy people and the migraine without aura group.

\begin{tabular}{|c|c|c|c|c|c|c|c|}
\hline \multirow{3}{*}{ Variables } & \multicolumn{6}{|c|}{ Genotype } & \multirow{3}{*}{ P value* } \\
\hline & \multicolumn{2}{|c|}{ CC } & \multicolumn{2}{|c|}{ CT } & \multicolumn{2}{|c|}{$\mathrm{TT}$} & \\
\hline & Count & (Percent) & Count & (Percent) & Count & (Percent) & \\
\hline Without aura & 47 & (49.5\%) & 45 & $(47.4 \%)$ & 3 & $(3.2 \%)$ & 0015 \\
\hline Control & 86 & (60.6\%) & 41 & (28.9\%) & 15 & (10.6\%) & 0.013 \\
\hline
\end{tabular}

\section{Discussion}

Given the multifactorial nature of migraine headache, investigations into the genetic aspects of this entity are always of interest. There are several neurological, hormonal and vascular genes that are considered as key factors in the pathophysiology of migraine [5]. It has been proposed that endothelial dysfunction, which increases oxidative stress and promotes inflammatory processes, is involved in the pathogenesis of migraine [6]. In this respect, MTHFR C677T genetic polymorphisms may play a crucial role in vascular diseases [3]. In MTHFR C677T polymorphism, a point mutation changes an alanine into a valine residue at Position 222 within the catalytic domain of the enzyme, which in turn reduces the enzymatic capacity by up to $50 \%$ [3].

In the present study, we evaluated the association between migraine and the TT genotype, as well as the T allele, of MTHFR polymorphism C677T in migraine patients who were referred to the Imam Hussein and Loghman-e-Hakim Hospitals (Tehran, Iran) between June 2016 and March 2017. Our findings demonstrate that there was no statistically significant difference regarding the TT genotype or T allele distributions between those in the migraine with aura group and control subjects. Meanwhile. the frequency of the TT genotype among those in the migraine without aura group was significantly lower than the controls and those in the migraine with aura group.

In 2000, it was reported for the first time that homozygous C677T mutation in the MTHFR gene is a genetic risk factor for migraine in Japanese patients [2]. This result was further supported by the observation that individuals with the C677T genotype have greater susceptibility towards migraine, while the prevalence of T allele has been found to be higher in Turkish [7] and Australian [8] migraineurs with aura. Furthermore, in a population-based sample to analyze the association between a specific genetic variant and a common migraine type, the TT genotype was associated with the prevalence of migraineurs with aura [9]. Moreover, migraine with aura was significantly more prevalent among the $\mathrm{T}$ allele carriers than CC genotype Russian patients [10]. In addition, it has been reported that the MTHFR genotype is associated with specific clinical variables of migraine, including unilateral head pain, physical activity discomfort and stress [11].

It has been proposed that an SNP in the MTHFR gene C677T causes mild hyperhomocysteinemia, which is associated with endothelial cell injury, reduced production of nitric oxide and increased oxidative stress, and may contribute to the activation of the trigeminal fibers [5]. Moreover, high plasma levels of homocysteine were related to a high risk of cardiovascular disease, cerebrovascular disease and peripheral vascular disease [12]. Since homocysteine derivatives act as NMDA receptor agonists, they enhance glutamatergic neurotransmission [10]. In addition, both in vivo and in vitro studies have demonstrated that moderate hyperhomocysteinemia produces neurotoxic effects $[13,14]$, which may be involved in the pathogenesis of migraine.

In contrast with these findings, other investigations have failed to confirm an association between MTHFR C677T polymorphism and migraine in Spanish [15], Finnish[16], Portuguese [17] and North Indian [18] migraineurs. Furthermore, meta-analysis studies have shown that there is no significant association between the MTHFR gene and migraine; only in migraine with aura is the TT genotype associated with a higher risk of disease compared with the CC genotype $[19,20]$.

In the Han Chinese population from the Fujian Province in Southern China, genotypic distribution of MTHFR C677T was significantly different in terms of a higher frequency of $T$ allele in migraineurs compared with controls. While no difference was found between patients who experience migraine with aura and controls, T allele frequency was significantly higher among migraineurs without aura than controls [21], which is in contrast to the findings of several previous reports. Furthermore, in an entirely different observation, Schurks et al. [22] reported that the MTHFR T677T genotype was associated with a reduced risk of migraine with aura, which only appeared in less than six attacks/ year. In parallel with our observation, Todt et al. [23] reported that there was no significant difference regarding $T$ allele or TT genotype distributions between 656 German migraine patients with aura and 625 healthy controls; however, they did not evaluate the frequency of the TT genotype among migraine patients without aura. Moreover, vividly contrasting with the results of the present study, Saeedi et al. [24] reported that MTHFR C677T polymorphism is a potential genetic risk factor for migraine attacks in patients, both with and without aura, referred to other hospitals in Tehran (Imam Khomeini and Rasool Akram Hospitals).

In 2013 Sadeghi et al. [25], evaluated homocysteine levels in 130 Iranian patients with migraine attacks. They did not find any significant relationship between homocysteine levels and characteristics of migraine attacks such as "severity, frequency, duration and headache diary result. A part of this discrepancy could be due to different sample sizes and different ethnicities. The sample size of the present study was also limited for the migraines with aura so this must be considered as a limitation of the present study. 


\section{Conclusion}

Since ethnicity has a role in the gene diversity of migraine, this study was designed to determine the role of MTHFR C677T gene polymorphism in Iranian patients who suffer from migraine headaches. Generally, our findings demonstrate that

\section{References}

1 Sutherland HG, Griffiths LR (2017) Genetics of migraine: Insights into the molecular basis of migraine disorders. Headache 57: 537-569.

2 Kowa H, Yasui K, Takeshima T (2000) The homozygous C677T mutation in the methylenetetrahydrofolate reductase gene is a genetic risk factor for migraine. Am J Med Genet 96: 762-764.

3 Frosst P, Blom HJ, Milos R (1995) A candidate genetic risk factor for vascular disease: A common mutation in methylenetetrahydrofolate reductase. Nat Genet 10: 111-113.

4 Van Der Molen EF, Arends GE, Nelen WLDM (2000) A common mutation in the 5,10-methylenetetrahydrofolate reductase gene as a new risk factor for placental vasculopathy. Am J Obstet Gynecol 182: $1258-1263$.

5 Maher BH, Griffiths LR (2011) Identification of molecular genetic factors that influence migraine. Mol Genet Genomics 285: 433-446.

6 Pizza V, Agresta A, Agresta A (2012) Migraine and genetic polymorphisms: An overview. Open Neurol J 6: 65-70.

7 Kara I, Sazci A, Ergul E (2003) A ssociation of the C677T and A1298C polymorphisms in the 5,10 methylenetetrahydrofolate reductase gene in patients with migraine risk. Molecular Brain Res 111: 84-90.

8 Lea RA, Ovcaric M, Sundholm J (2004) The methylenetetrahydrofolate reductase gene variant $\mathrm{C677T}$ influences susceptibility to migraine with aura. BMC Med 2: 3.

9 Scher Al, Terwindt GM, Verschuren WMM (2006) Migraine and MTHFR C677T genotype in a population-based sample. Ann Neurol 59: 372-375.

10 Azimova JE, Sergeev A V, Korobeynikova LA (2013) Effects of MTHFR gene polymorphism on the clinical and electrophysiological characteristics of migraine. BMC Neurol 13: 1.

11 Liu A, Menon S, Colson NJ (2010) Analysis of the MTHFR C677T variant with migraine phenotypes. BMC Res Notes 3:213.

12 Di Minno MND, Tremoli E, Coppola A (2010) Homocysteine and arterial thrombosis: Challenge and opportunity. Thromb Haemost 103: 942-961.

13 Lipton Sa, Kim WK, Choi YB (1997) Neurotoxicity associated with dual actions of homocysteine at the N-methyl-D-aspartate receptor. Proc Natl Acad Sci USA 94: 5923-5928.
MTHFR C677T polymorphism, which is responsible for reducing MTHFR activity in the folate metabolism, is not a major genetic susceptibility factor for migraine in the investigated population. Therefore, further elucidation is required to define whether MTHFR C677T polymorphism is associated with susceptibility towards migraine in the Iranian population.

14 Zieminska E, Lazarewicz JW (2006) Excitotoxic neuronal injury in chronic homocysteine neurotoxicity studied in vitro: The role of NMDA and group I metabotropic glutamate receptors. Acta Neurobiol Exp 66: 301-309.

15 Oterino A, Valle N, Bravo Y (2004) MTHFR T677 homozygosis influences the presence of aura in migraineurs. Cephalalgia 24:491-494.

16 Kaunisto MA, Kallela M, Hamalainen E, Kilpikari R, Havanka $H$, et al. (2006) Testing of variants of the MTHFR and ESR1 genes in 1798 Finnish individuals fails to confirm the association with migraine with aura. Cephalalgia 26: 1462-1472.

17 Ferro A, Castro MJ, Lemos C (2008) The C677T polymorphism in MTHFR is not associated with migraine in Portugal. Dis Markers 25: 107-113.

18 Joshi G, Pradhan S, Mittal B (2009) Role of the ACE ID and MTHFR C677T polymorphisms in genetic susceptibility of migraine in a north Indian population. J Neurol Sci 277: 133-137.

19 Rubino E, Ferrero M, Rainero I (2009) Association of the C677T polymorphism in the MTHFR gene with migraine: A meta-analysis. Cephalalgia 29: 818-825.

20 Schürks M, Zee RYL, Buring JE, Kurth T (2010) MTHFR 677C $>T$ and ACE D/I polymorphisms and migraine attack frequency in women. Cephalalgia 30: 447-456.

21 An XK, Lu CX, Ma QL (2013) Association of MTHFR C677T polymorphism with susceptibility to migraine in the Chinese population. Neurosci Lett 549: 78-81.

22 Schürks M, Rist PM, Kurth T (2010) MTHFR 677C>T and ACE D/I polymorphisms in migraine: A systematic review and meta-analysis. Headache 50: 588-599.

23 Todt U, Freudenberg J, Goebel I (2006) MTHFR C677T polymorphism and migraine with aura. Ann Neurol 60: 621-622.

24 Saeedi S, Owji A, Ansari M (2015) MTHFR gene polymorphisms and susceptibility to migraine attacks. AMLS 1: 61-66.

25 Sadeghi O, Maghsoudi Z, Askari G, Khorvash F, Feizi A (2014) Association between serum levels of homocysteine with characteristics of migraine attacks in migraine with aura. J Res Medical Sci: The Official JRMS 19: 1041- 1045. 